Supporting information for:

\title{
Amphiphilic phospholipid-based riboflavin derivatives for targeting nanomedicines
}

\author{
Nataliia Beztsinna ${ }^{1}$, Yoannna.Tsvetkova ${ }^{2}$, Matthias Bartneck ${ }^{3}$, Twan Lammers ${ }^{2}$, Fabian \\ Kiessling ${ }^{2}$, Isabelle Bestel ${ }^{1}$ \\ 1 MMBE team, UMR 5248 CBMN, Bordeaux University, Pessac, France \\ 2 Experimental Molecular Imaging, RWTH Aachen University Clinic, Aachen, Germany \\ 3 Gastroenterology and Metabolic Disorders, RWTH Aachen University Clinic, Aachen, Germany
}

\section{Table of contents}

Figure S1. ${ }^{1} \mathrm{H}-\mathrm{NMR}$ spectrum of RfdiC14 (compound 10).

Figure S2. ${ }^{31} \mathrm{P}-\mathrm{NMR}$ spectrum of RfdiC14 (compound 10).

Figure S3. ${ }^{13} \mathrm{C}-\mathrm{NMR}$ spectrum of RfdiC14 (compound 10).

Figure S4. HMQC, 2D-NMR spectrum of RfdiC14 (compound 10).

Figure S5. ${ }^{1} \mathrm{H}$ NMR spectrum of compound $\mathbf{8}$.

Figure S6. ${ }^{13} \mathrm{C}$ NMR spectrum of compound 8 .

Figure S7. ESI-MS spectrum of compound 4.

Figure S8. ESI-MS spectrum of compound 8.

Figure S9. ESI-MS spectrum of RfdiC14 (compound 10).

Figure S10. Characterization of compound 11 (DSPE-PEG-RF)

Figure S11. Calibration curves for riboflavin and rhodamine B

Figure S12. Calibration of LCL in $\mu \mathrm{CT} / \mathrm{FMT}$ phantom.

Figure S13. Morphology and stability characterization of control and targeted liposomes.

Figure S14. MTT cytotoxicity assay results.

Figure S15. Various steps of $\mu \mathrm{CT} / \mathrm{FMT}$ organ segmentation.

Figure S16. Biodistribution LCL in vivo. 
NMR spectra

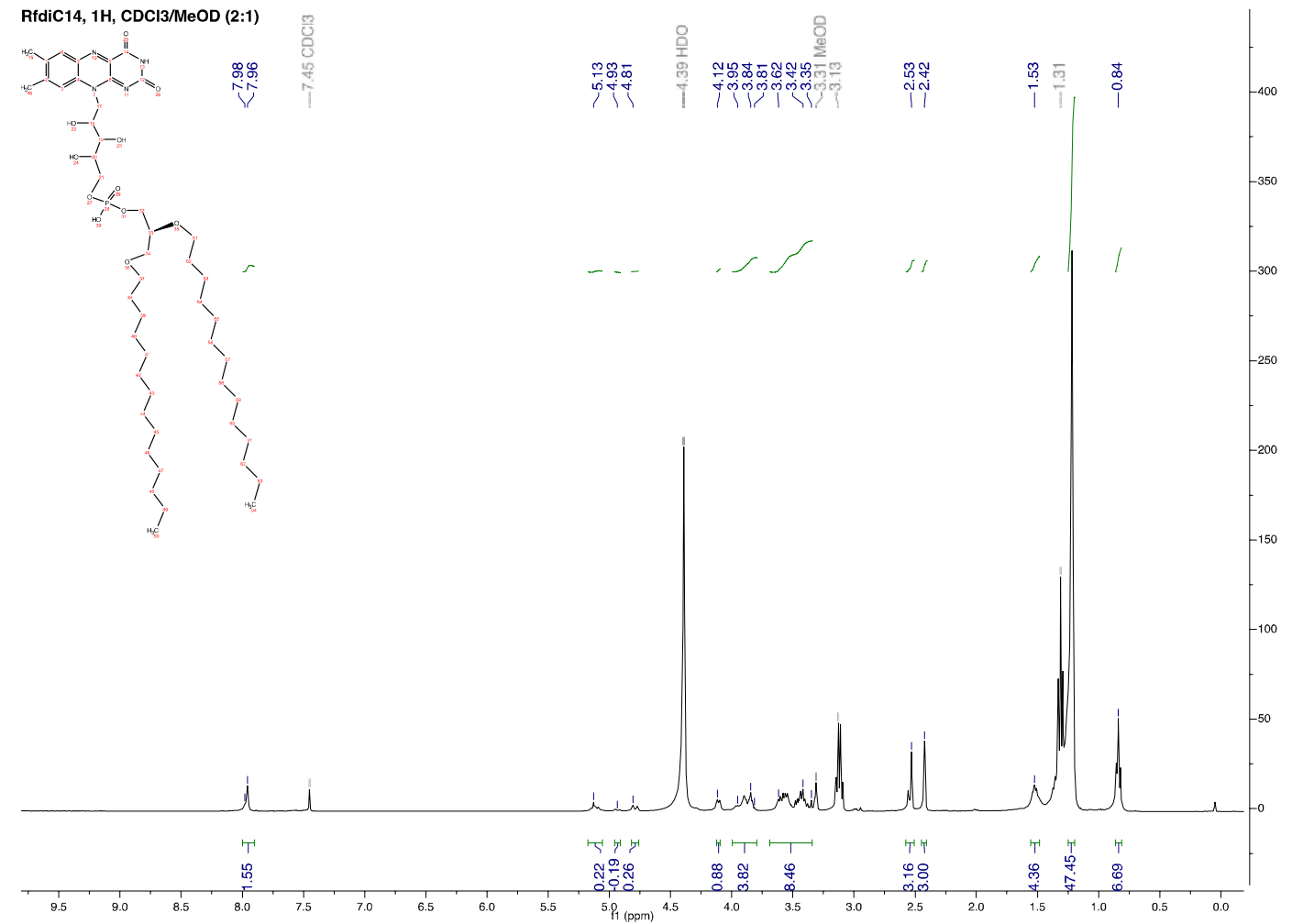

Figure S1. ${ }^{1} \mathrm{H}-\mathrm{NMR}$ spectrum of RfdiC14 (compound 10).

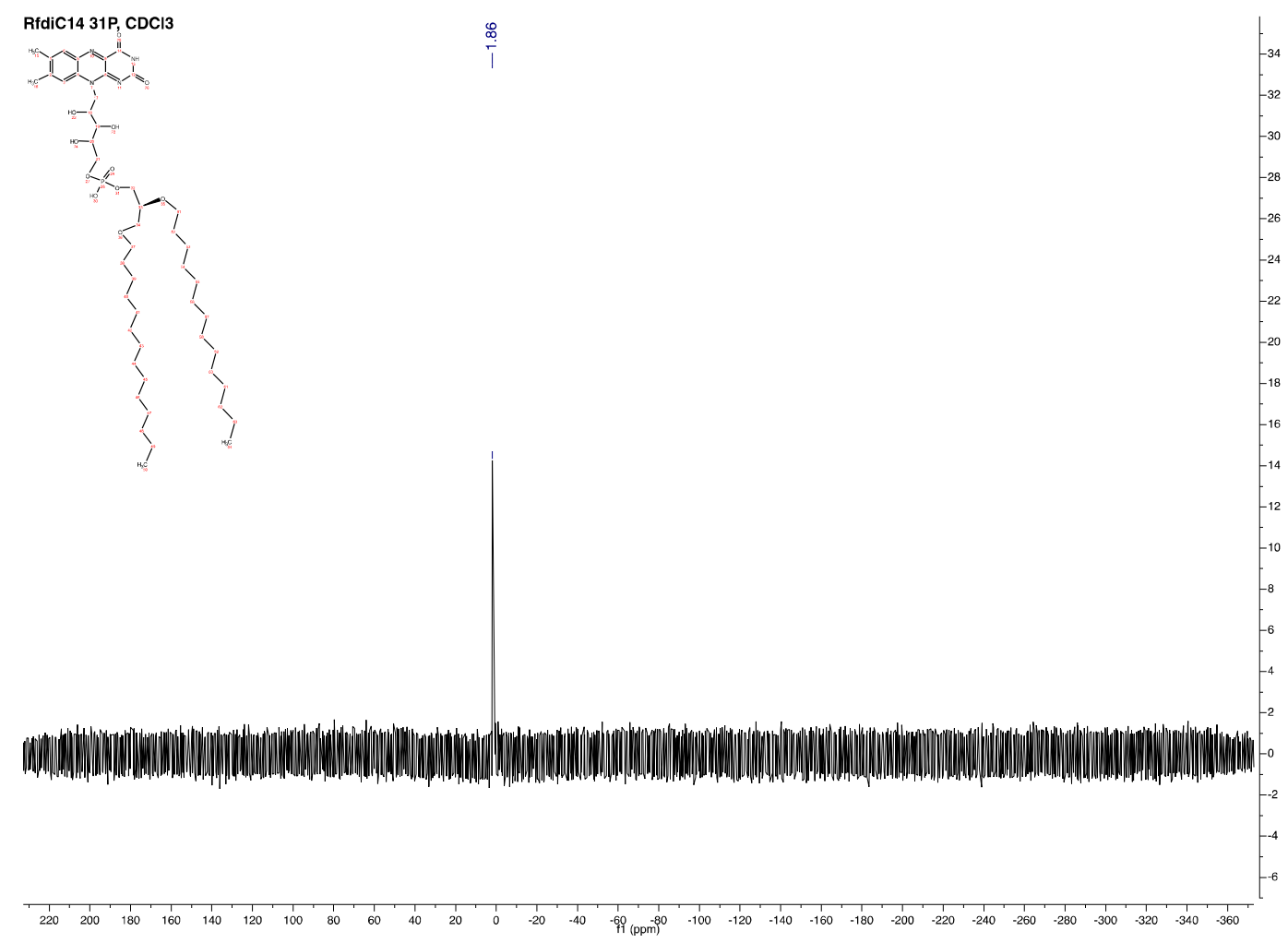

Figure S2. ${ }^{31} \mathrm{P}-\mathrm{NMR}$ spectrum of RfdiC14 (compound 10). 


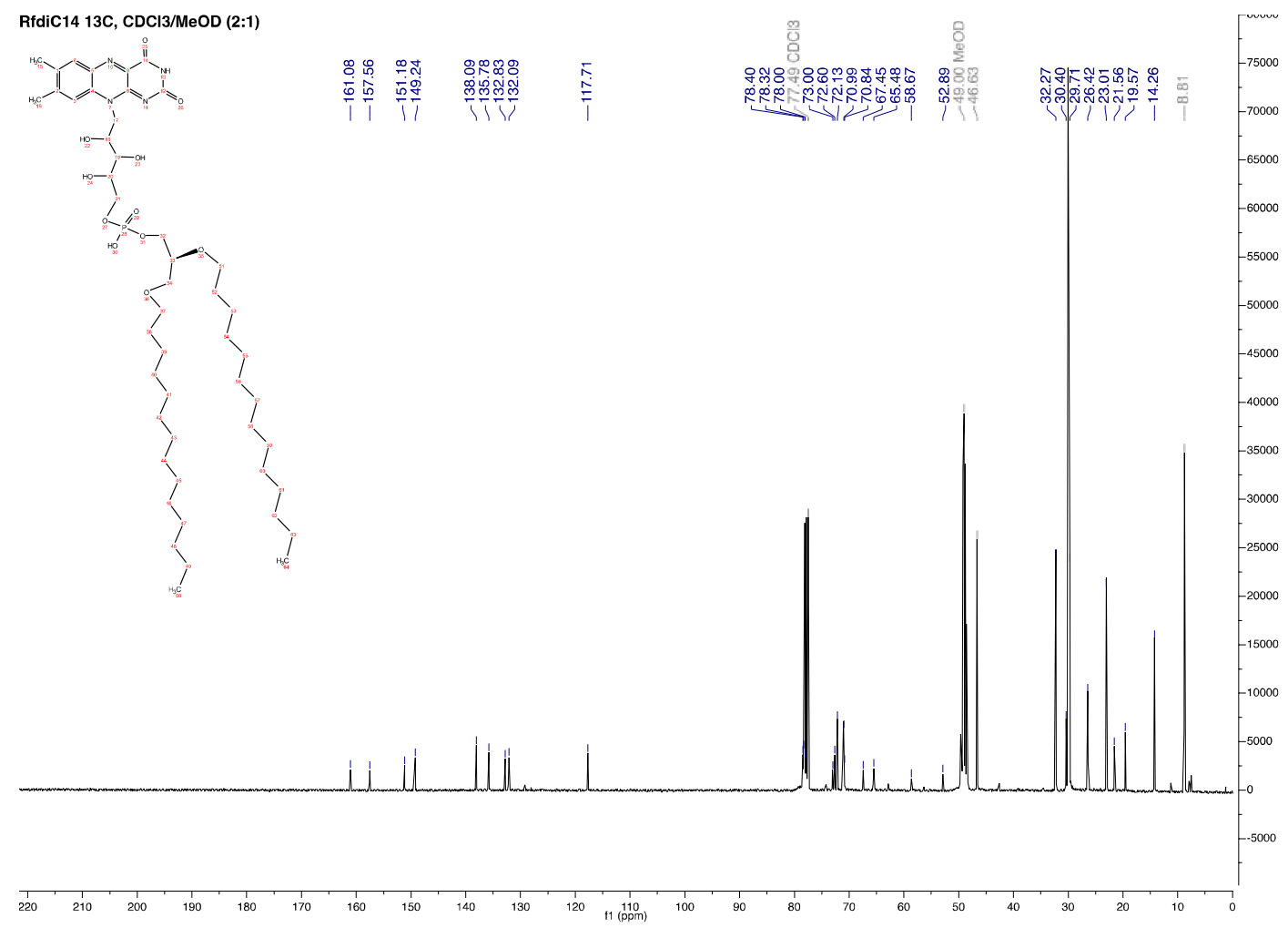

Figure S3. ${ }^{13} \mathrm{C}$-NMR spectrum of RfdiC14 (compound 10).

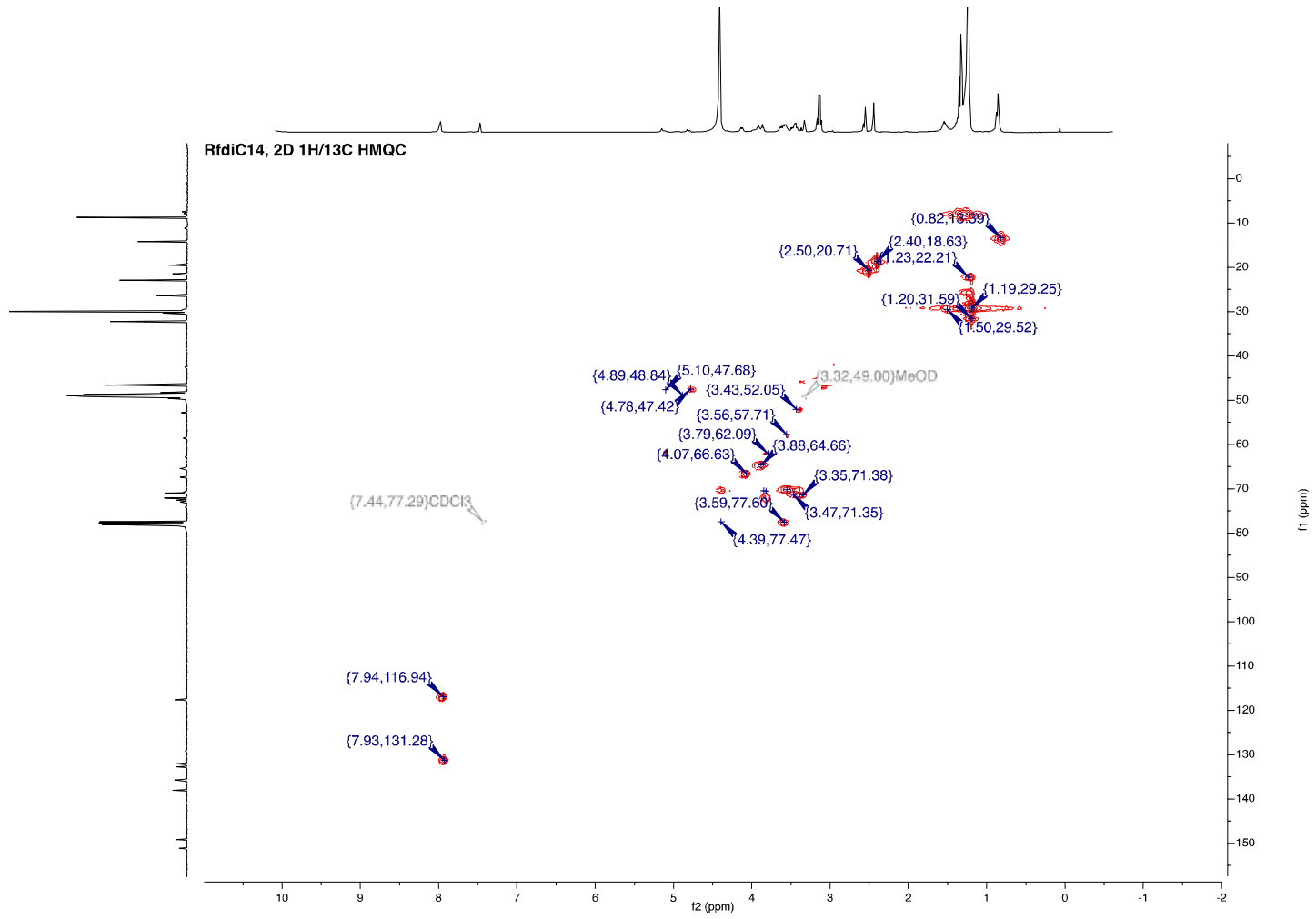

Figure S4. HMQC, 2D-NMR spectrum of RfdiC14 (compound 10). 


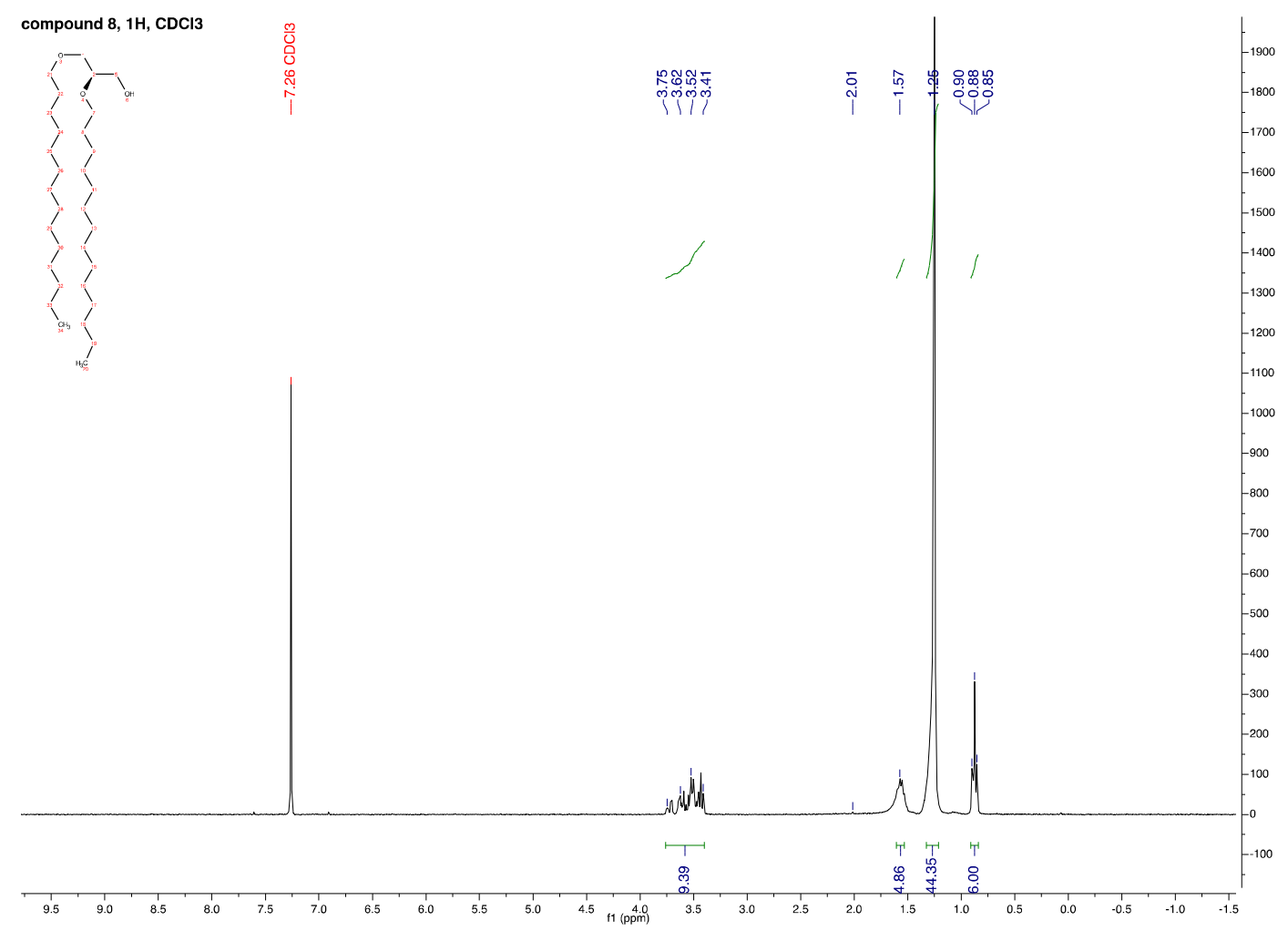

Figure S5. ${ }^{1} \mathrm{H}$ NMR spectrum of compound 8.

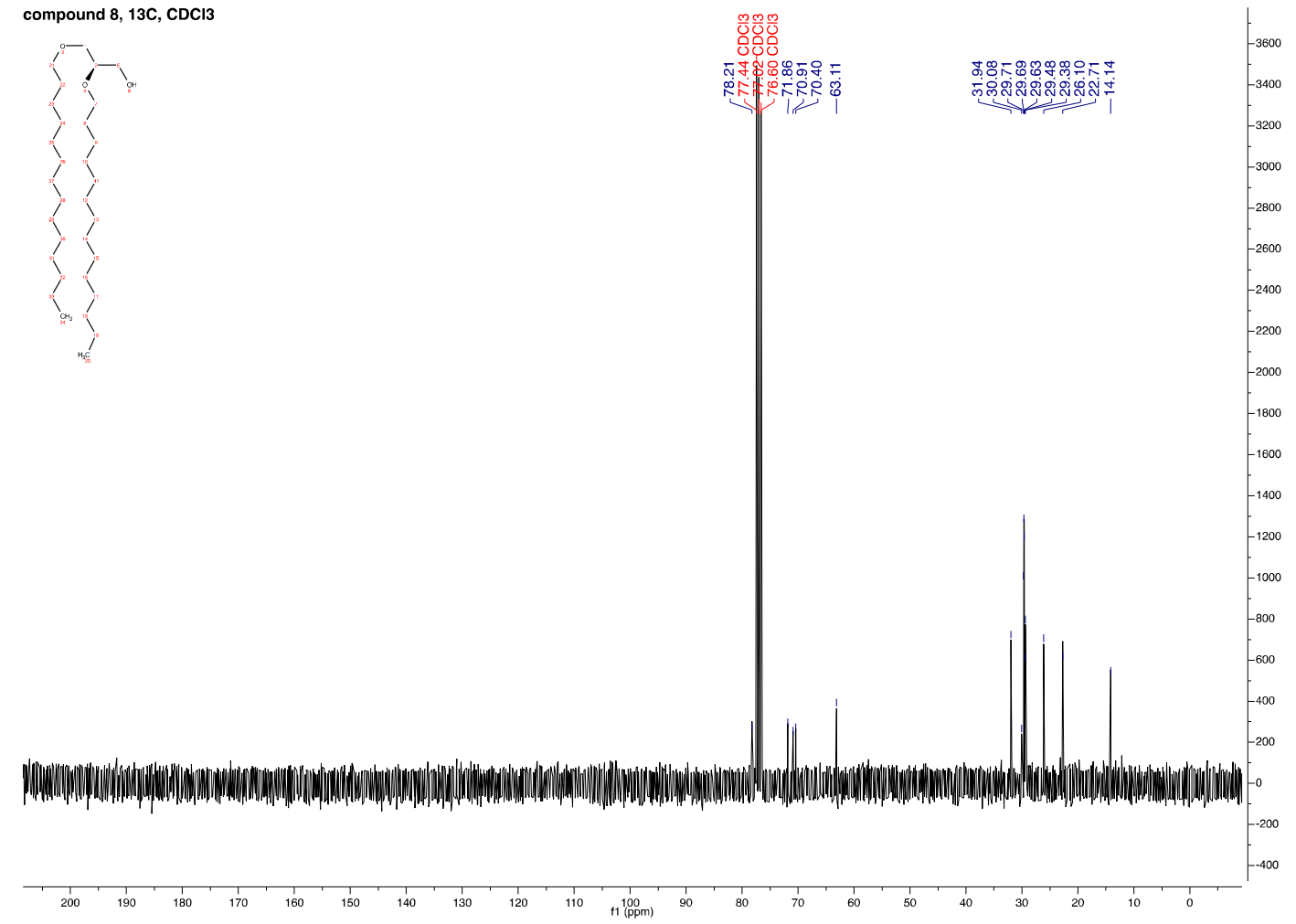

Figure S6. ${ }^{13} \mathrm{C}$ NMR spectrum of compound 8 . 
ESI-MS spectra

140221-Exac-1411-IB-NB-NB18-6neg \#1-68 RT: 0.06-1.50 AV: 68 NL: 1.31E6 T: FTMS - p ESI Full ms [100.00-2000.00]

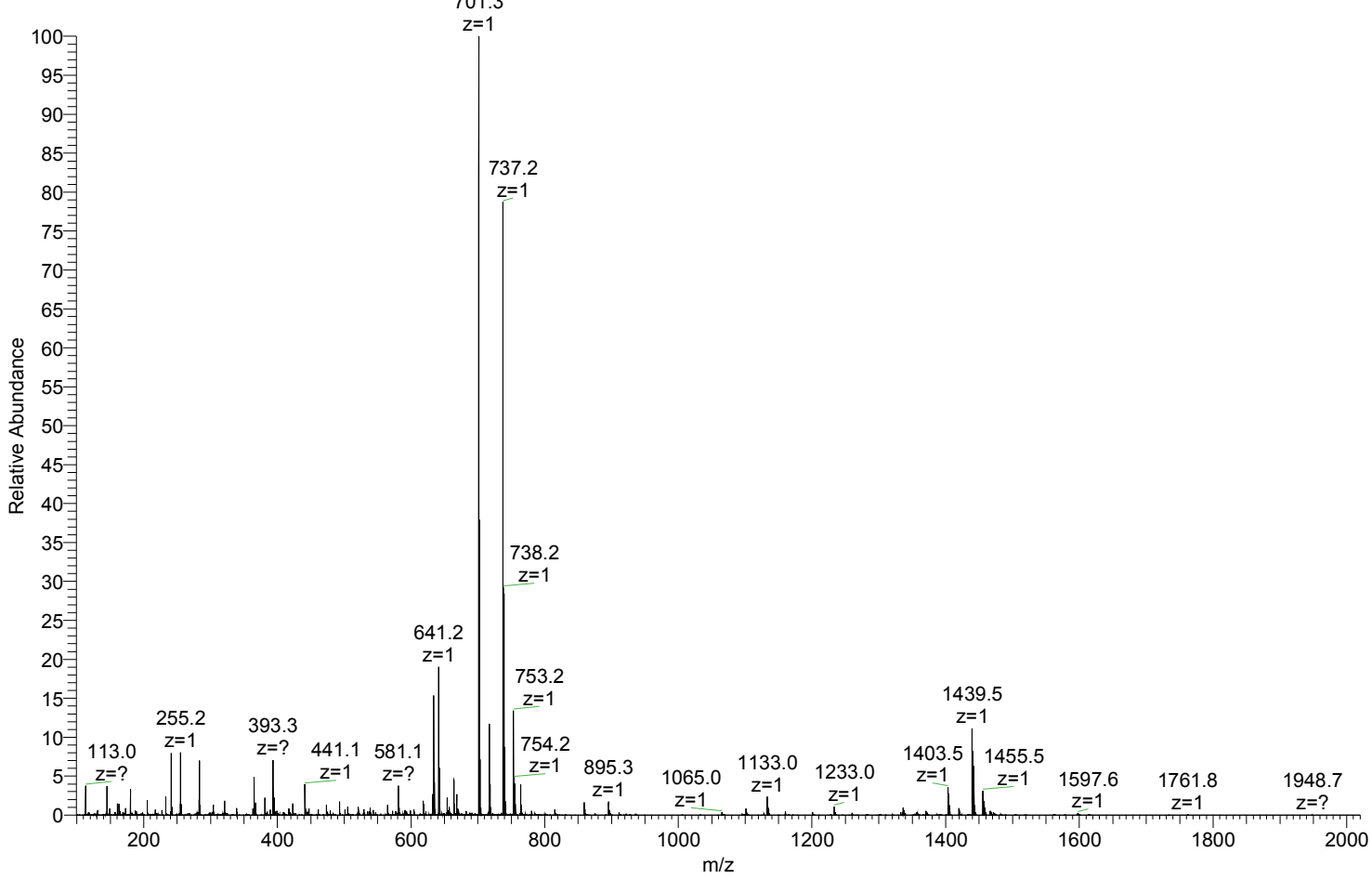

Figure S7. ESI-MS spectrum of compound 4.

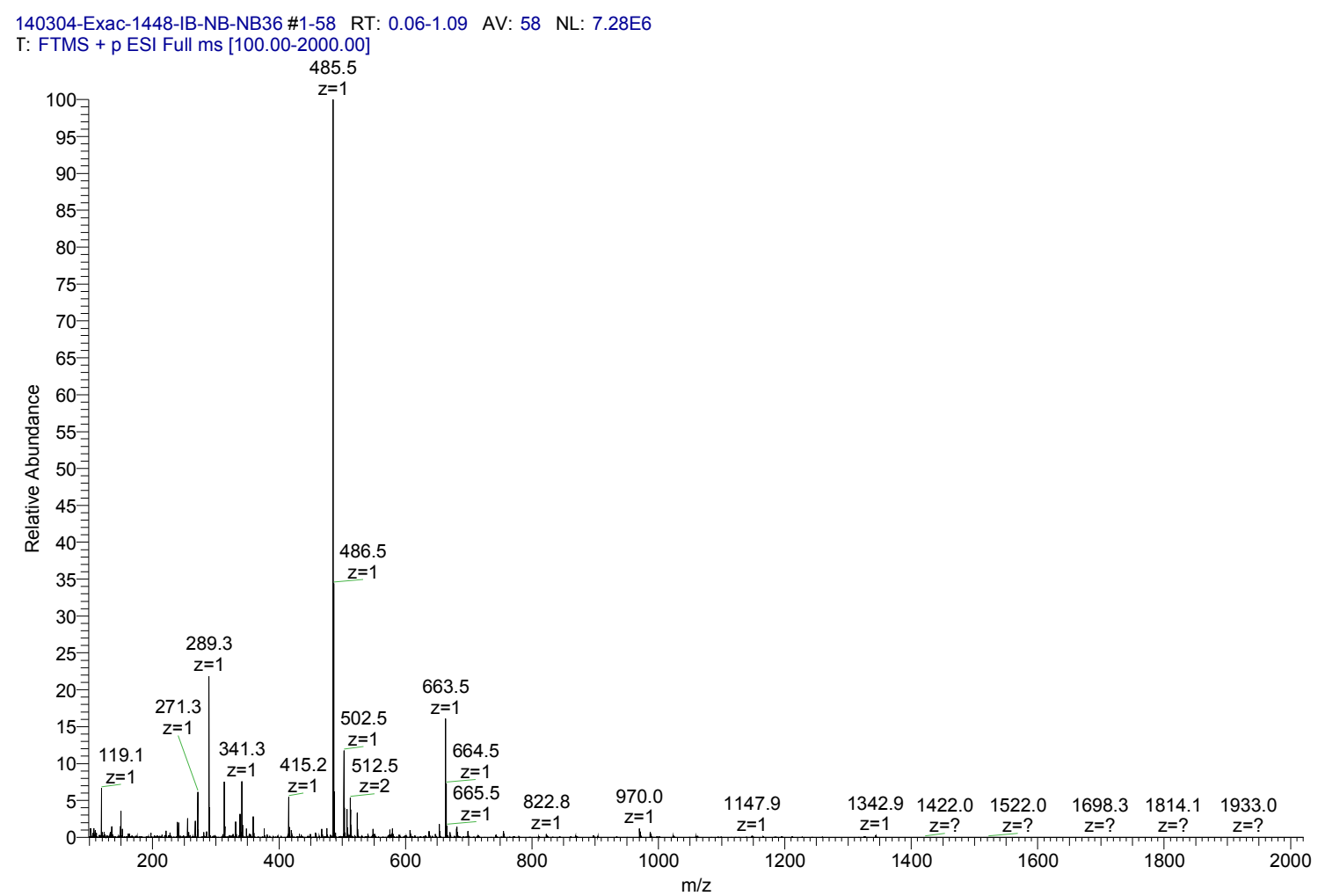

Figure S8. ESI-MS spectrum of compound 8. 
140422-t-xac-15/6-IB-NB-NBbU \#1-18 KI: 0.04-1.3/ AV: 18 NL: 2.68t6 T: FTMS + p ESI Full ms [100.00-2000.00]

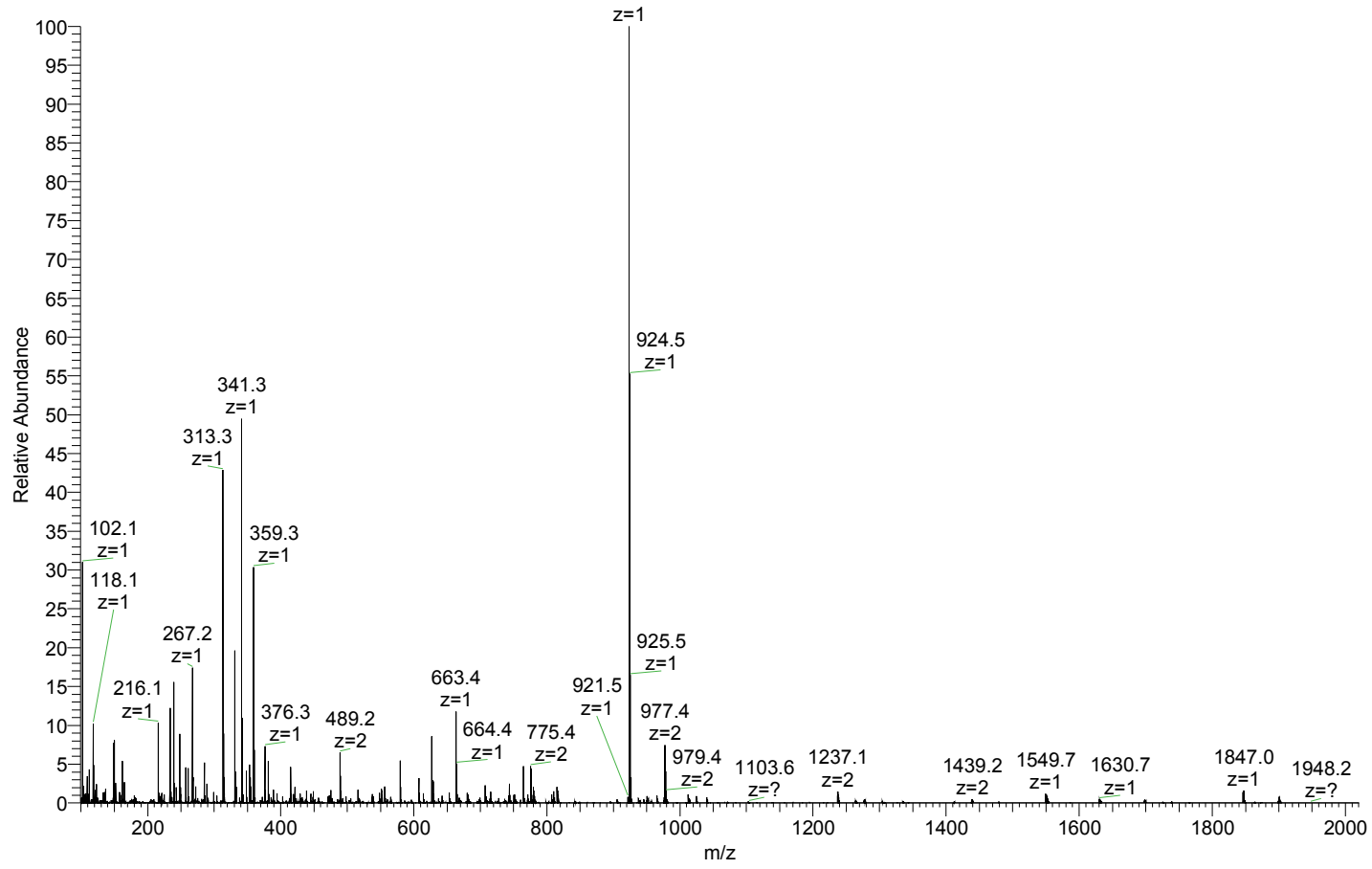

Figure S9. ESI-MS spectrum of RfdiC14 (compound 10). 
A

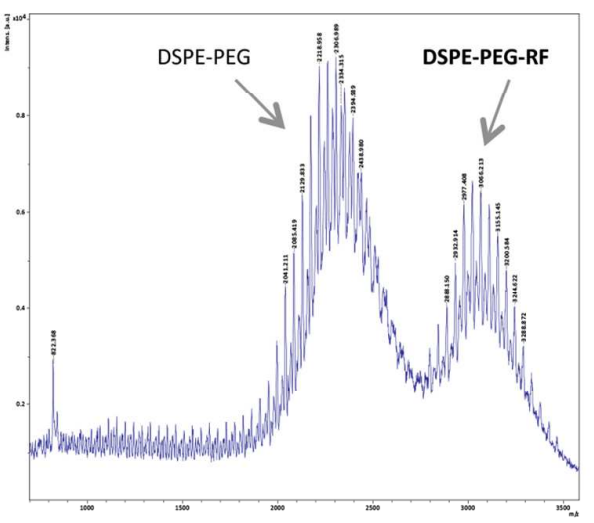

B

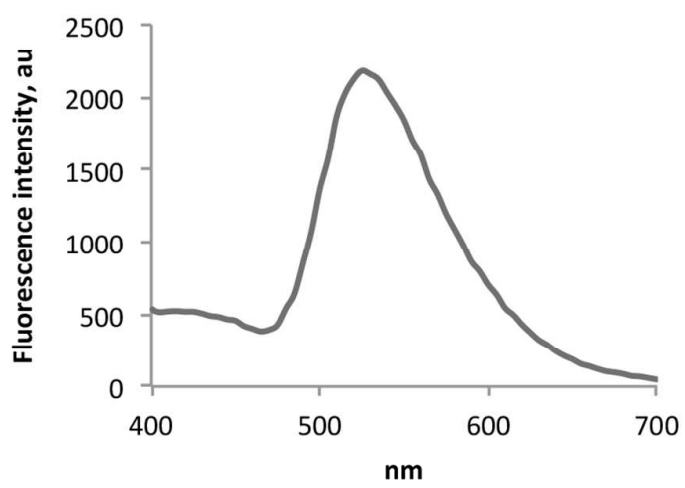

C

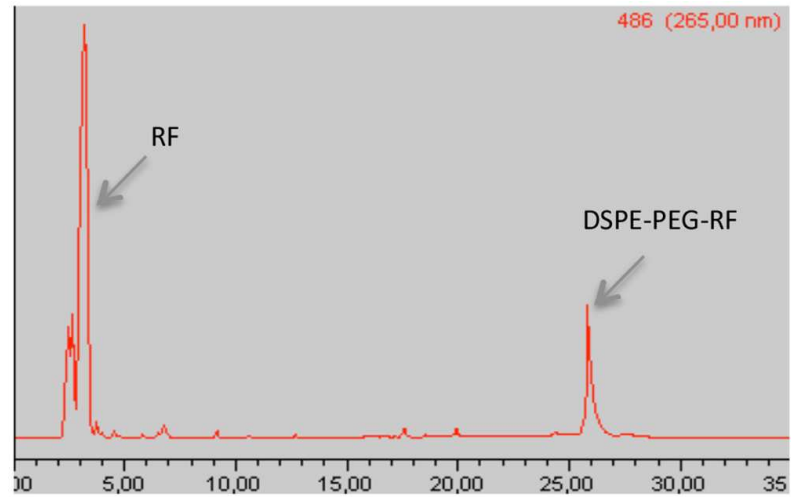

Figure S10. Characterization of compound 11 (DSPE-PEG-RF): A. MALDI mass spectrum of DSPE-PEG-RF; B. Fluorescence emission spectrum of DSPE-PEG-RF; C. Reverse-phase HPLC chromatogram of DSPE-PEG-RF synthesis. 

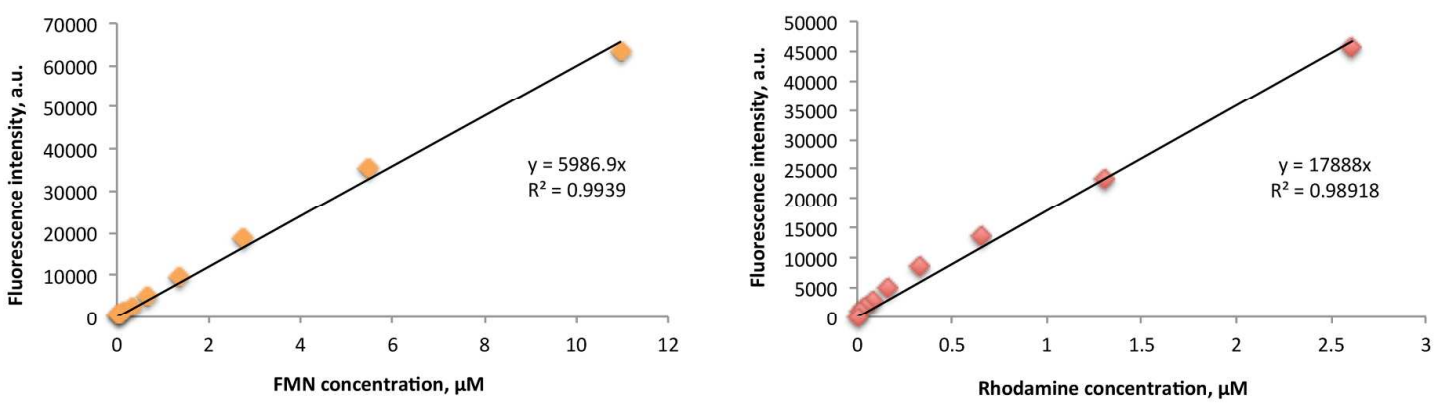

Figure S11. Calibration curves for riboflavin (left) and rhodamine B (right) in buffer.
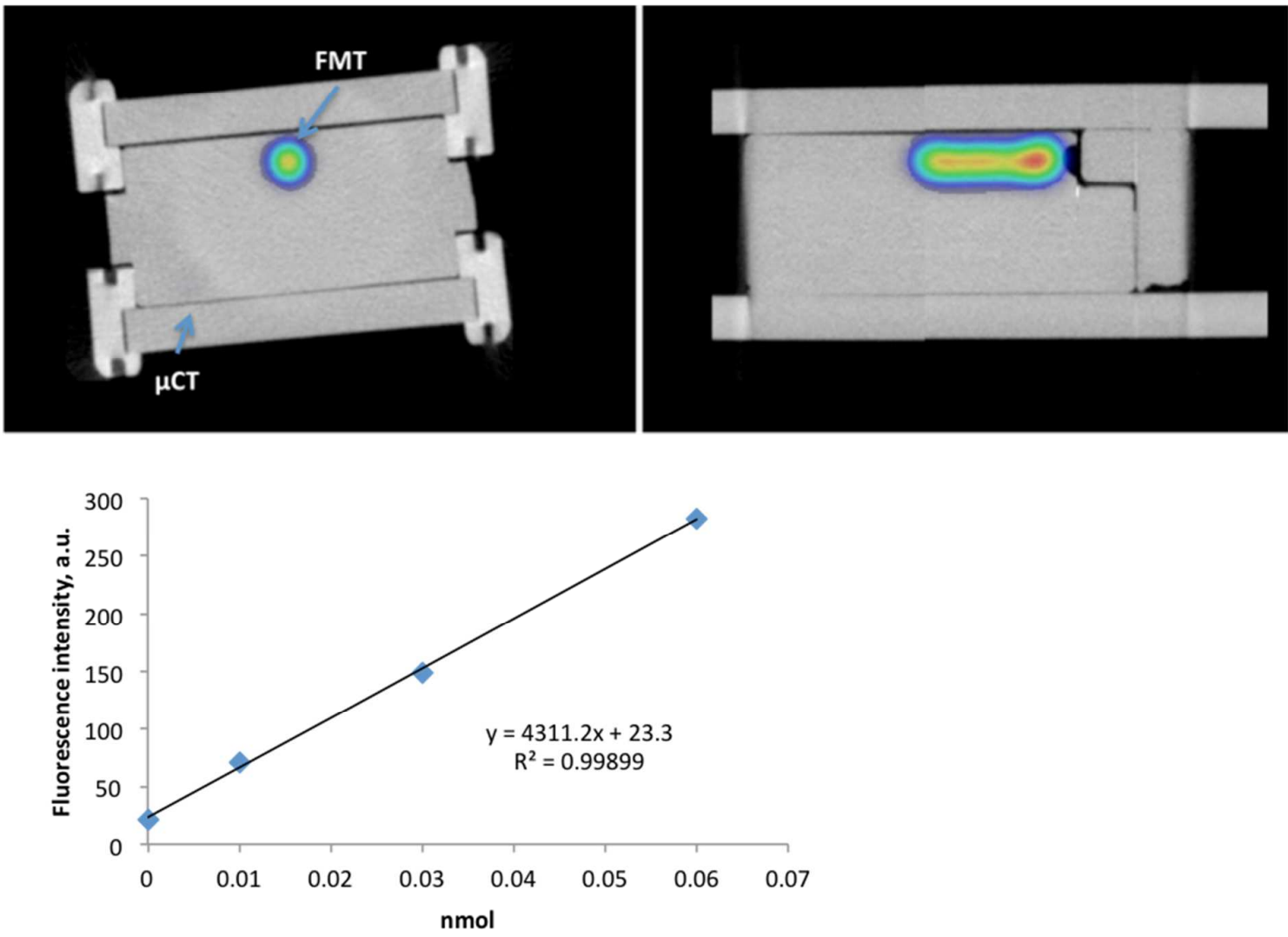

Figure S12. Up and side view of phantom image obtained from $\mu \mathrm{CT}$ and FMT fusion (up); $\mu \mathrm{CT} / \mathrm{FMT}$ study of DiR liposomes in phantom, fluorescence (ex. $750 \mathrm{~nm}$ ) signal intensities are plotted against DiR liposomes quantity (nmol) (down). The linear dependence between DiR labelled LCL and the emitted fluorescence, described by the following equation - $y=$ $4311.2 x+23.3\left(\mathrm{R}^{2}=0.99238\right)$, where $y$ represents the fluorescence signal intensity and $x-$ the quantity of DiR in nmol. 
A

B

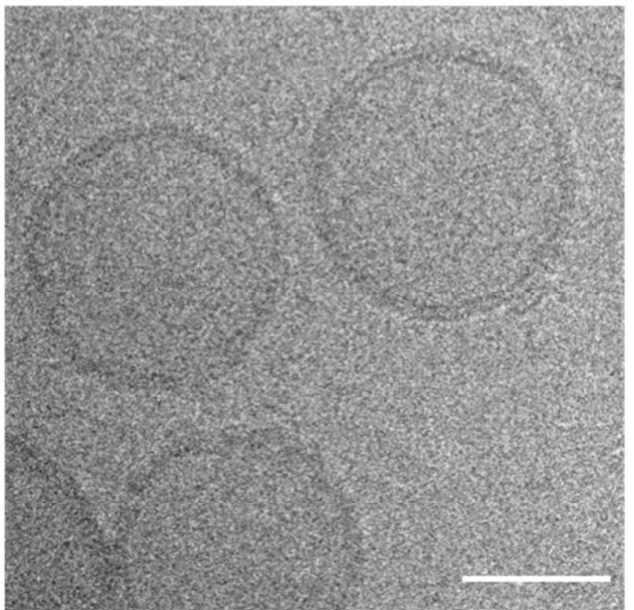

C

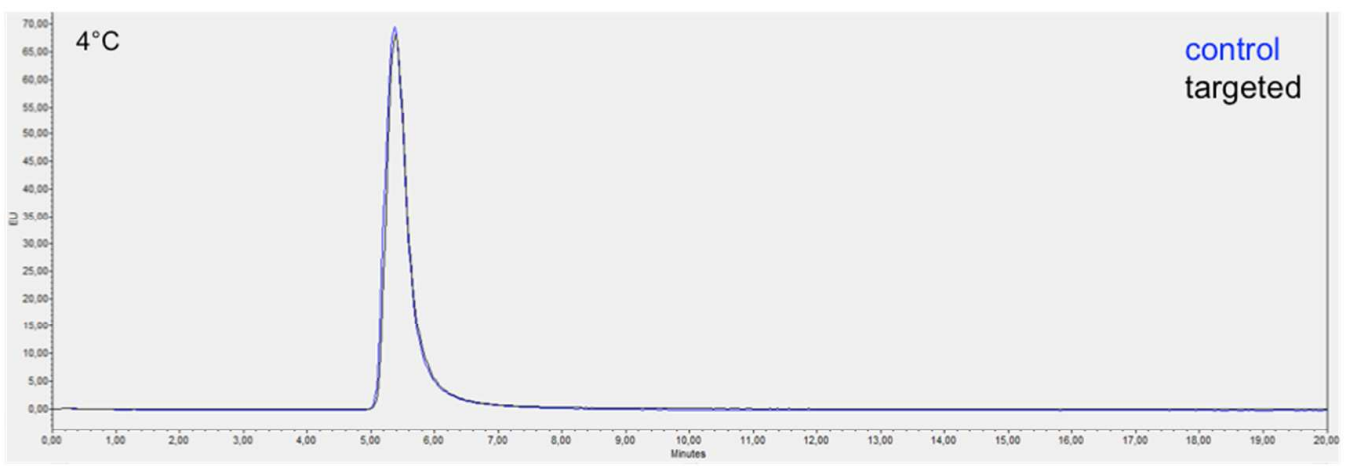

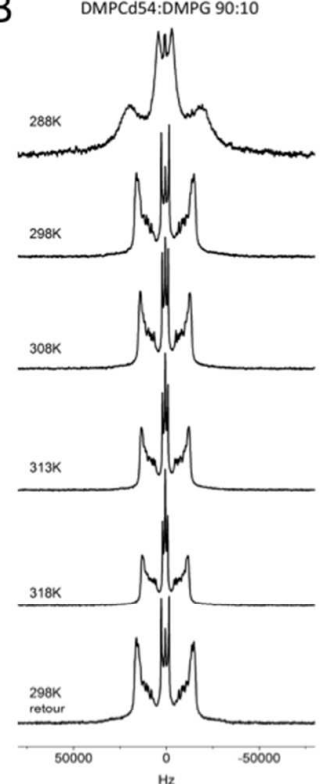

DMPCd54:DMPG:Rfdic14 90:10:10

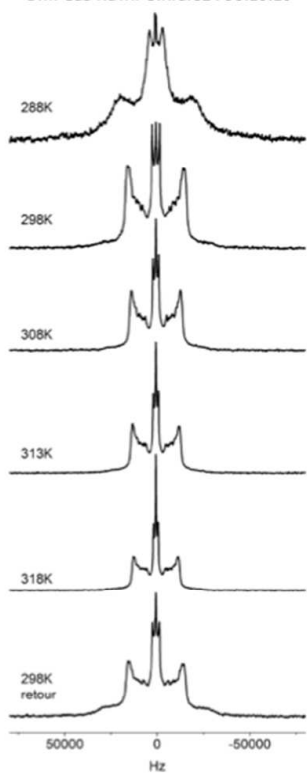

control control targeted

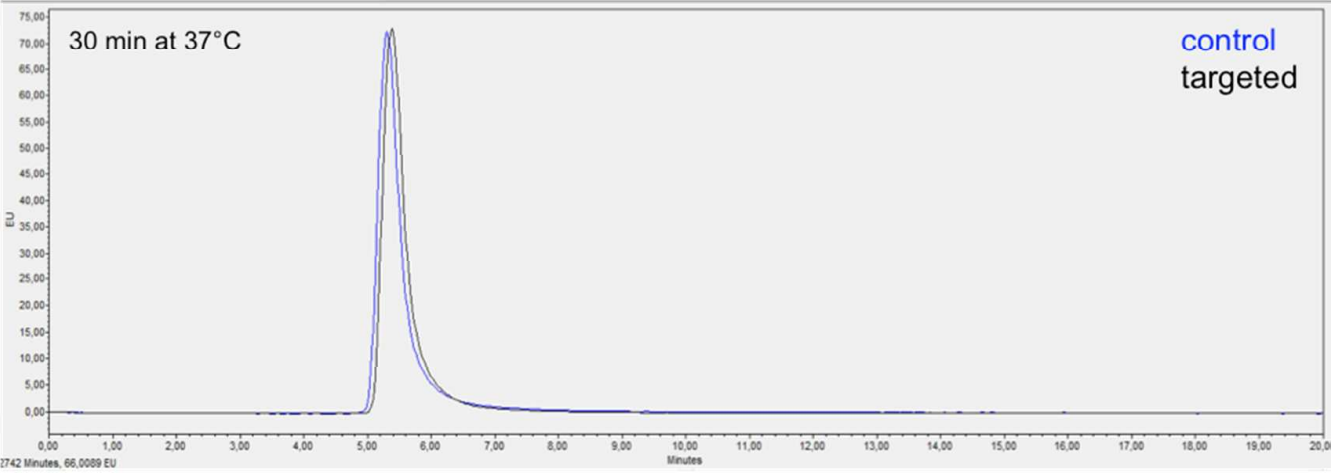

Figure S13. Morphology and stability characterization of control and targeted liposomes: A. Cryo-EM photograph of targeted liposomes; scale bar $50 \mathrm{~nm}$. B. ${ }^{2} \mathrm{H}-\mathrm{NMR}$ spectra of DMPCd54: DMPG (left) and DMPCd54:DMPG:RfdiC14 (right); C. GPC profile of control and targeted liposomes at storage conditions $\left(4^{\circ} \mathrm{C}\right)$ and after 30 min heating at $37^{\circ} \mathrm{C}$, control formulation is depicted in blue and targeted is in black. 

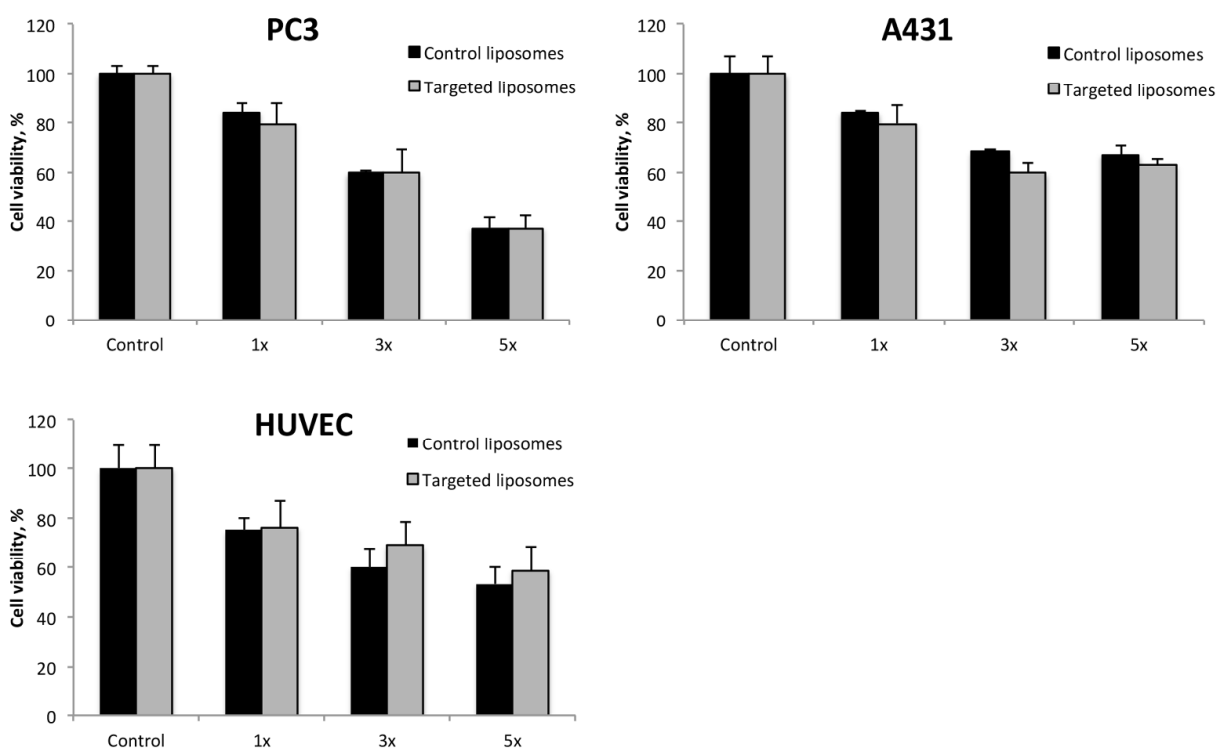

Figure S14. MTT cytotoxicity assay results. PC3 and A431 cells as well as HUVEC were incubated with standard (50 nM or 1x) and higher concentration (150 nM and $250 \mathrm{nM}$ for $3 \mathrm{x}$ and $5 \mathrm{x}$, respectively) of targeted and control liposomes; the values are presented as mean \pm $\mathrm{SD}$ from $\mathrm{n}=3$ experiments. 


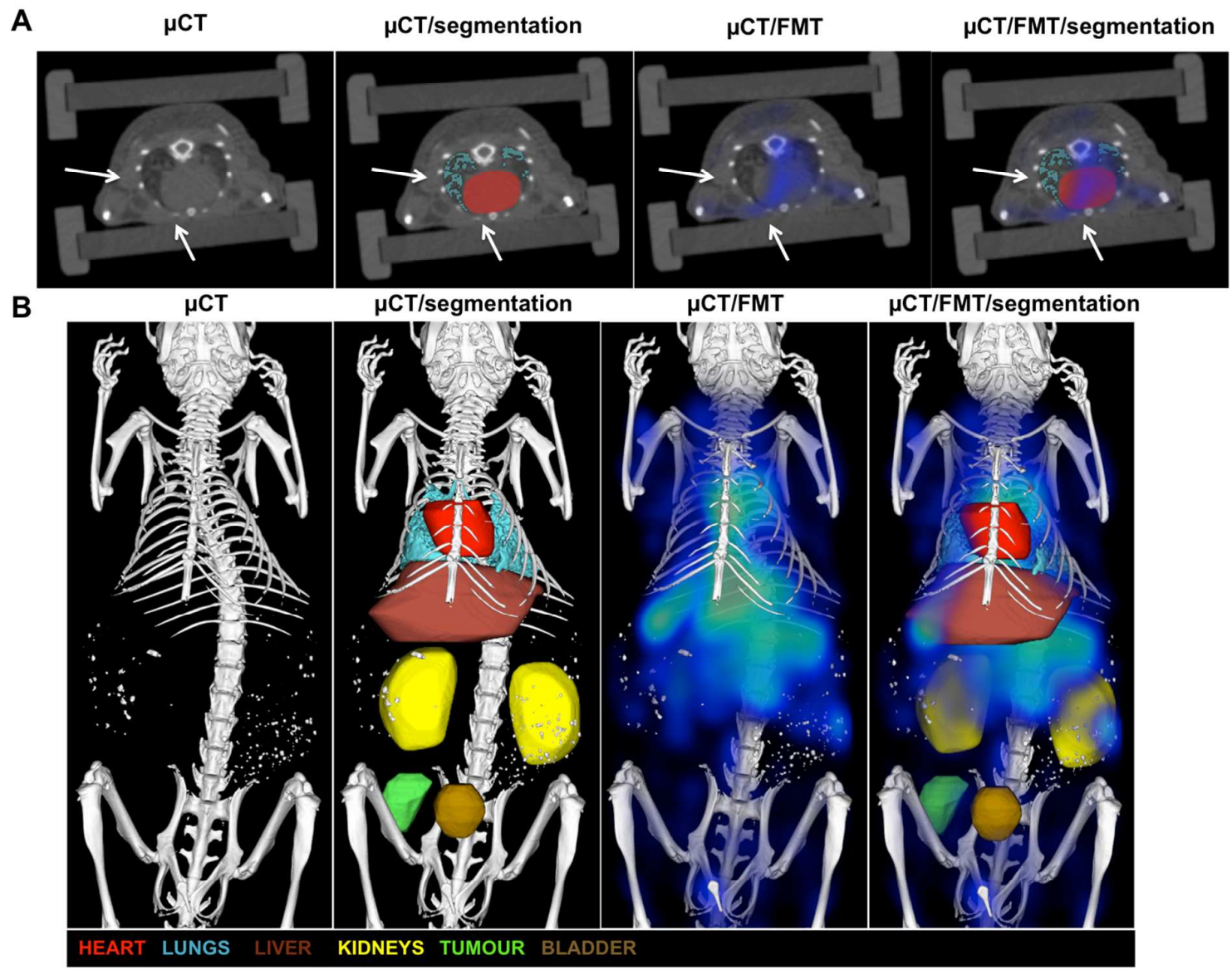

Figure S15. A. Various steps of organ segmentation in transverse view of a mouse; heart (red) and lungs (light blue) are marked with white arrows. $\mu \mathrm{CT}$ contrast is grey and fluorescent signal appears in a shape of dark blue cloud; B. Various steps of organ reconstruction and fluorescence quantification in 3D. Segmented organs are presented in various colours: heart - red, lungs - light blue, liver - brown, kidneys - yellow, tumour green, bladder - beige. Fluorescent signal appears in the form of diffuse blue clouds. 

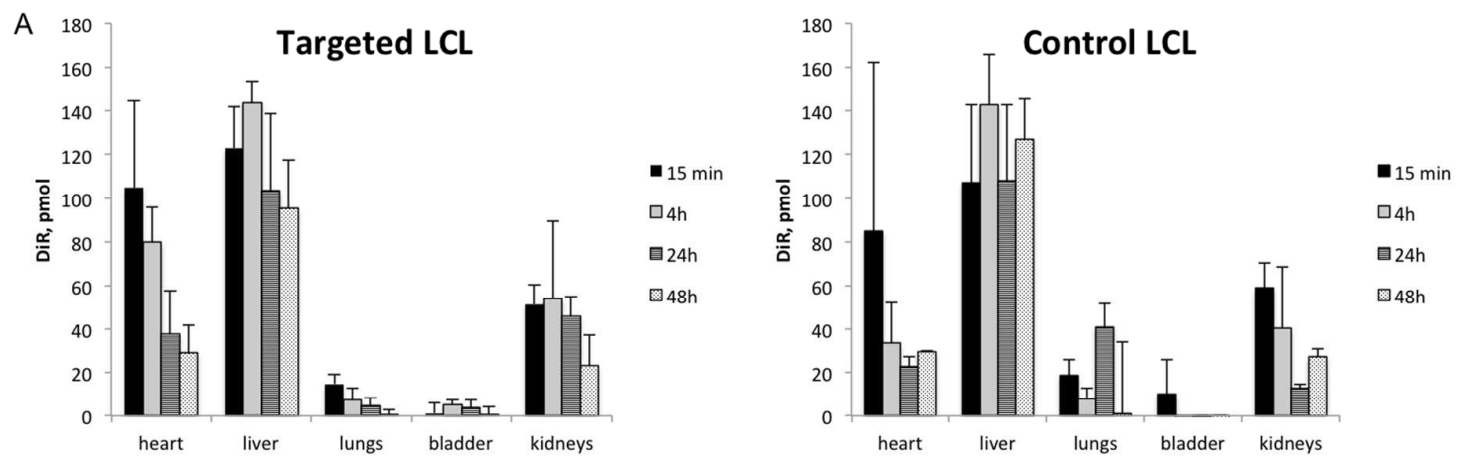

B

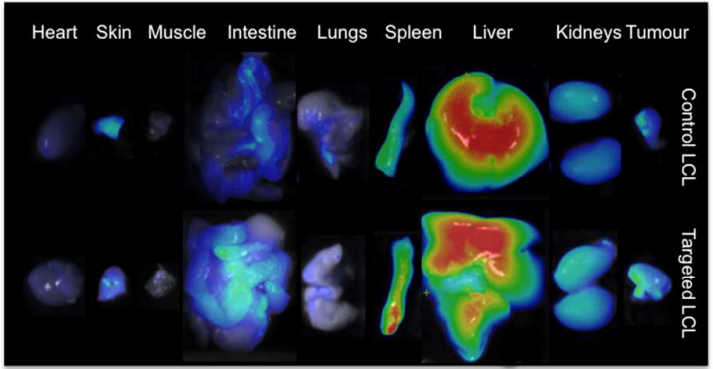

Figure S16. Biodistribution of LCL in vivo: A. Longitudinal 3D fluorescence quantification in healthy organs after i.v. injection of control or targeted LCL; B. ex vivo 2D fluorescence reflectance images of various organs and $\mathrm{PC} 3$ tumour xenografts from control and targeted group. Results presented as average $\pm \mathrm{SD}$ from $\mathrm{n}=3$ mice per group, $\mathrm{SD}-$ standard deviation. 